who had at least one full sibling born in the same period $(\mathrm{N}=1381436)$. All individuals were followed from age 28 years until the age of death, emigration, or December 2009. Using Cox' regression analyses, we estimated HRs for mortality according to educational status.

Results Both conventional cohort analyses and intra sibling analyses were carried out. Educational differences observed in the cohort analyses were attenuated in the intra- sibling analyses.

Conclusion The attenuation of the association in the intra sibling analyses indicates that environment in childhood and/or genetic setup explain some of the association between educational status and mortality. However, significant associations still persisted in intra-sibling analyses, supporting an independent effect of education on mortality.

\section{P1-338 THE ASSOCIATION OF MATERIAL DEPRIVATION AND RR OF INFANT MORTALITY IN PENINSULAR MALAYSIA}

doi:10.1136/jech.2011.142976f.30

F Soofen,* A A Jemain, W Z W Zin. Universiti Kebangsaan Malaysia, Selangor,
Malaysia

The aim of this paper is to investigate whether areas with greater deprivation index have higher RR of infant mortality in Peninsular Malaysia. This investigation is essential for the regional planning and development by policy makers to reduce the inequality and health gap across region within the country. We begin this investigation by studying the census based data for year 1991 and 2000 for the 81 administrative districts. These data are then correlated with RR of infant mortality data for the period of 1990-2000. In this paper, Bayesian hierarchical method is used to rank area deprivation and RR of infant mortality for each administrative district. The results are then mapped through choropleth maps to see the association between the two risk factor and health indicator. The analysis showed that regional material deprivation is strongly associated with the RR of infant mortality.

\section{P1-339 PATTERN OF DROWNING AND ITS BURDEN IN NORTH COAST OF IRAN}

doi:10.1136/jech.2011.142976f.31

H Soori,* S Akbarpour, N Jafari, A Khosravi, E Ainy. Safety Promotion and Injury Prevention Research Center, Shahid Beheshti University of Medical Sciences, Tehran, Iran

Background and objective Drowning is a neglected serious public health problem in the northern provinces of Iran. Since the first step for health program planning in each community health is the priority setting, in this study, the epidemiological pattern was reviewed and burden of drowning was calculated.

Methods This study used data on death registration system and deaths due to drowning disruption in two provinces of Mazandaran and Gilan happened in 2008 were addressed. Initially with the help of descriptive statistics to describe characteristics of drowning, using the standard WHO forms the burden of drowning was calculated. Results During 2008, 158 indigenous people from the provinces died from drowning. Of these 140 cases $(88.6 \%)$ were male and 18 patients $(11.4 \%)$ were female. The mean age of the drowned was $26.4(\mathrm{SD}=16.2)$ years. The drowning death rate was 2.9 per 100000 population in two provinces. In examining place and time, most cases (85.4\%) occurred in summer, the month of August (29.7\%). Number of years lost was 4110 equivalent of 76.1 per 100000 respectively. Most disabled age lost life years (DALYs) was seen to age group 10-19 years.
Conclusion With regard to the issue that most drowned in the sea and occurred in the summer time and the highest number of DALYs was in the age group 10 to 19 years, these findings need to be considered for prevention strategies in these provinces.

\section{P1-340 FOLATE STATUS AND FOLIC ACID INTAKE 6 YEARS FOLLOWING MANDATORY FORTIFICATION OF FLOUR IN BRAZIL: A POPULATION-BASED STUDY IN SÃO PAULO}

doi:10.1136/jech.2011.142976f.32

${ }^{1} \mathrm{~J}$ Steluti, ${ }^{*} \mathrm{~V}$ T Baltar, ${ }^{1} \mathrm{R}$ S Bigio, ${ }^{1} \mathrm{R}$ M Fisberg, ${ }^{1} \mathrm{D} \mathrm{M}$ L Marchioni. ${ }^{1}$ Department of Nutrition, Faculty of Public Health, University of Sao Paulo, Sao Paulo, Sao Paulo, Brazil; ${ }^{2}$ Department of Epidemiology, Faculty of Public Health, University of Sao Paulo, Sao Paulo, Sao Paulo, Brazil

Introduction To prevent neural tube defects (NTD) folic acid fortification of wheat and corn flour has been mandatory in Brazil since 2004. The purpose of this study was to examine the effect that folic acid fortification has had on serum folate levels and its contribution of total folate intake.

Methods Data were obtained from two $24 \mathrm{~h}$ dietary recalls from a population-based study (2008-2010) in São Paulo-Brazil for 297 participants who also had serum folate concentrations measured. Folate intake was obtained using the software program Nutrition Data System for Research. Descriptive statistics, Pearson's correlates, Kruskal-Wallis and Student's t-tests were performed using Stata (Version 10.0)

Results The reference range (5th-95 th percentiles) for the population after the introduction of folic acid fortification was $4.6-16.4 \mathrm{ng} / \mathrm{ml}$ for serum folate. The prevalence of low $(<3 \mathrm{ng} / \mathrm{ml})$ serum folate was $0.6 \%$, while $1.4 \%$ of population exceed the limit value of $20 \mathrm{ng} / \mathrm{ml}$. Synthetic folate (folic acid) contributed $51.3 \%$ [49.2 to $53.495 \% \mathrm{CI}$ ] of total folate intake. Both natural folate and synthetic folate did not correlate with folate serum concentrations ( $p>0.05)$, however participants in the highest serum folate tertile were older $(p=0.001)$, and synthetic folate intake among adults and the elderly was less than the group aged $<19$ years $(p=0.01)$.

Conclusion There was a very low risk of folate deficiency in the studied population; older participants had higher levels of serum folate and lower intake of synthetic folate. Participants with excessive serum folate concentrations after the folic acid mandatory fortification of flour were identified.

\section{P1-341 DENGUE SPATIAL RISK DISTRIBUTION IN CAMPINAS, BRAZIL}

doi:10.1136/jech.2011.142976f.33

H G Malavasi, C Stephan, ${ }^{*}$ M R D Cordeiro. State University of Campinas, Faculty of Medical Sciences, Campinas, São Paulo, Brazil

Introduction Dengue fever is an arboviruse of great importance in public health. Currently, the urban population modus vivendi favours transmission and maintenance of breeding sites of the vector, Aedes aegypti. Given the complexity of vector control, epidemiological surveillance for early case detection is of fundamental importance for the prevention of outbreaks of major proportions. This research aims to study the spatial distribution of the risk of dengue in the city of Campinas from January to December 2007.

Methods Spatial RR of dengue fever was calculated for each 4 weeks in Campinas using generalised additive model in a case-control study. All 9018 reported and confirmed autochthonous dengue fever cases of residents in the municipality were geocoded, while controls were generated from a representative sample of the population of Campinas based on IBGE census. 
Results Maps show the temporal evolution and spatial distribution of dengue fever risk on the territory. Highest risk areas coincide with those of greater movement of people and lack of infrastructure in the municipality.

Conclusion The method was suitable for identifying risk areas, in which government's actions should be efficient and constant.

\section{P1-342 EMPIRICAL ANALYSIS OF THE RISK ESTIMATORS IN SPATIAL CASE-CONTROL STUDIES USING DIFFERENT SAMPLE DESIGNS}

doi:10.1136/jech.2011.142976f.34

${ }^{1} \mathrm{~A}$ C C N Mafra, ${ }^{2} \mathrm{C}$ T Codeco, ${ }^{1} \mathrm{R}$ Cordeiro, ${ }^{1} \mathrm{C}$ Stephan, ${ }^{* 1} \mathrm{~L}$ B Nucci. ${ }^{1}$ State University of Campinas, Campinas, São Paulo, Brazil; 'Fundação Oswaldo Cruz, Rio de Janeiro, Brazil

Introduction There are many sample designs for case-control studies. Three of them were simulated to investigate the properties of their risk estimators when the aim of the study is to analyse the space along with other covariates. They are: the case-base sampling, where all controls are selected at the beginning while the cases are sampled during the study as they occurs; the survivor sampling, in which both cases and controls are sampled at the end of the study; and the risk-set sampling, where both cases and controls are sampled during the study.

Methods A realistic at risk population was created by sampling individuals from the empirical spatial distributions derived from governmental census information of a Brazilian city. Two epidemic scenarios were built, a transmissible and a nontransmissible disease. We used the generalised additive models to estimate the risks in each different study, fitting semiparametric models with the geographical coordinates and other covariates as age, income, gender and study.

Results The results suggest that the estimated spatial risks are similar in the three sample designs, but the standard deviations vary in the space and, the widest variation occurs in the survivor sampling (for the nontransmissible disease) and in the case-base sampling (for the transmissible disease). The parametric estimates that are closest to the initially defined were attained by the risk-set sampling, at the nontransmissible disease scheme.

Conclusion We conclude that the best risk estimates are attained by sampling the controls at the same time of the cases, as the epidemic occurs.

\section{P1-343 GENDER DIFFERENCES IN HOME SMOKING RESTRICTIONS IN HUNGARY}

doi:10.1136/jech.2011.142976f.35

${ }^{1} \mathrm{~L}$ Nagymajtényi, ${ }^{1}{ }^{1} \mathrm{~A}$ Maróti-Nagy, ${ }^{2} \mathrm{D}$ Easterling, ${ }^{3} \mathrm{~T}$ Rogers, ${ }^{1} \mathrm{E}$ Paulik. ${ }^{1}$ Department of Public Health, Faculty of Medicine, University of Szeged, Szeged, Hungary; ${ }^{2}$ Wake Forest University School of Medicine, Winston-Salem, USA; ${ }^{3}$ Public Health Institute, Oakland, USA

This publication was made possible by Grant Number 1 R01 TW007927-01 from the Fogarty International Center, the National Cancer Institute, and the National Institutes on Drug Abuse, within the National Institutes of Health (NIH). Its contents are solely the responsibility of the authors and do not necessarily represent the official view of the NIH.

Introduction The relationship between the environmental tobacco smoke exposure and the increased risk of illness and death from a wide range of diseases is well-known. The aim of this study was to assess the connection between gender and home smoking restrictions.

Methods The first wave of a quantitative longitudinal study was delivered in 2009. A sample of individuals $(n=2250)$ aged
16-70 years completed a self-administered questionnaire (response rate $=57.4 \%$ ). Logistic regression models were applied to reveal the predictors of having a smoke-free home in smokers and non-smokers. Results Nearly two-third of people $34.9 \%$ of smokers and $71.4 \%$ of non-smokers) lived in homes where smoking was totally restricted. The predictors of having a smoke-free home were gender (female: $\mathrm{OR}=1.60 ; \mathrm{p}<0.0001$ ) and education (medium: $\mathrm{OR}=1.73$; high: $\mathrm{OR}=1.96 ; \mathrm{p}<0.001$ ) among non-smokers; while education (medium: $\mathrm{OR}=1.81$; high: $\mathrm{OR}=1.3 .20 ; \mathrm{p}<0.001)$ and living in the rural area $(\mathrm{OR}=1.91 ; \mathrm{p}<0.0001)$ in smokers.

Conclusion Home smoking restrictions were associated with gender and education among non-smokers, with education and living place among smokers. The results showed that greater attention must be placed on non-smoker males, on smokers living in urban areas, and in general on low educated people to improve tobacco control in Hungary.

\section{P1-344 CORONARY ARTERY LESIONS OF INCOMPLETE KAWASAKI DISEASE: THE NATIONWIDE SURVEY OF 2007-2008 IN JAPAN}

doi:10.1136/jech.2011.142976f.36

${ }^{1} \mathrm{D}$ Sudo, ${ }^{*} \mathrm{Y}$ Monobe, ${ }^{1} \mathrm{M}$ Yashiro, ${ }^{1} \mathrm{M}$ Mieno, ${ }^{1} \mathrm{R}$ Uehara, ${ }^{2} \mathrm{~K}$ Tsuchiya, ${ }^{2} \mathrm{~T}$ Sonobe, ${ }^{1} Y$ Nakamura. ${ }^{1}$ Jichi Medical University, Shimotsuke, Japan; ${ }^{2}$ Japanese Red Cross Medical Center, Shibuya-ku, Japan

Objectives To observe the recent epidemiologic features of incomplete Kawasaki disease (KD) patients reported to the Japanese nationwide survey, and to compare risk factors for developing coronary artery lesions (CALs) between incomplete $\mathrm{KD}$ and complete $\mathrm{KD}$.

Methods In the 2007-2008 nationwide survey, $23337 \mathrm{KD}$ patients were reported and then classified by their number of principal symptoms for analyses. In this study, complete $\mathrm{KD}$ was defined as having five or more of the six principal symptoms; incomplete $\mathrm{KD}$ as having four or fewer regardless of the presence or absence of CALs.

Results Compared with complete KD patients, younger ages at first hospital visit, a higher incidence rate of CALs, less frequency of both initial and additional intravenous immunoglobulin (IVIG) administration, later IVIG administration, less total dose of IVIG administration, and less frequency of steroid therapy were observed among those with incomplete $\mathrm{KD}$. Also particularly in patients having CALs, the same delayed and insufficient IVIG therapy was observed in incomplete $\mathrm{KD}$. Multivariate analysis showed that the risk factors for CALs observed in incomplete $\mathrm{KD}$ were almost the same as those observed in complete $\mathrm{KD}$, except for receiving initial IVIG therapy. The initial IVIG administration was risk of developing CALs in incomplete $\mathrm{KD}$, but not risk rather protective in complete $\mathrm{KD}$.

Conclusions The results imply the existence of a common pathological basis in generating CALs regardless of presentation types of $\mathrm{KD}$. Therefore, more timely diagnosis and treatment to incomplete $\mathrm{KD}$ patients could lead to the further prevention of cardiac lesions caused by $\mathrm{KD}$

\section{P1-345 REGULATORY RISK ASSESSMENT IN EPIDEMIOLOGY: PRINCIPLES AND METHODOLOGY}

doi:10.1136/jech.2011.142976f.37

Y Sun, ${ }^{*}$ F Bochmann. Institute for Occupational Safety and Health of German Social Accident Insurance (IFA), Sankt Augustin, Germany

Regulatory risk assessment is a useful tool in the development of public policy, regulation and decision making in occupational safety and health. It focuses on a detailed quantification of occupational 